\title{
Le Véloce-Sport et
l'invention de la culture
cycliste
}

\author{
Martin Hurcombe \\ Professor \\ University of Bristol \\ United Kingdom \\ M.J.Hurcombe@bristol.ac.uk
}

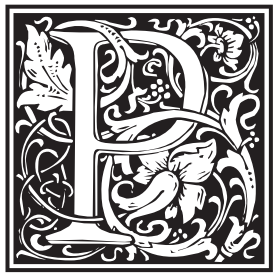

ublié pour la première fois à Bordeaux en mars 1885, l'hebdomadaire Le Véloce-Sport se transforma dans l'espace de quelques années en un organe national avant de disparaître en novembre 1897 alors que le cyclisme était en plein essor. Envisagé dans ses origines comme la voix du Véloce Club Bordelais (VCB) (Dauncey, 2012 : 22), il se convertit très rapidement en centre de recrutement pour l'Union Vélocipédique Française (UVF), une première tentative nationale de rassembler les clubs cyclistes français dans le but de protéger leurs intérêts et de centraliser le sport en France (Poyer, 2004 : 299300). Le Véloce-Sport avait donc une double mission : au niveau régional, celle de promouvoir les valeurs du VCB et de servir les intérêts de ses membres ; au niveau national, celle d'attirer d'autres clubs vélocipédiques dans l'orbite de l'UVF. C'est pour cette raison que les historiens du cyclisme français l'ont parfois exploité comme source et comme matière premières sans pour autant s'intéresser à sa pratique journalistique, à la diversité de son contenu, ni à sa contribution générale à ce que l'on pourrait nommer la culture cycliste de la fin-de-siècle (Holt, 2014). Une lecture plus attentive du Véloce-Sport offre le spectacle d'une culture sportive en voie de développement et illustre donc le rôle fondamental du journalisme sportif, ainsi que celui de ses lecteurs, dans la construction de cette culture. Ainsi arriverons-nous à une meilleure connaissance de la part du journalisme sportif de la fin du XIX ${ }^{\mathrm{e}}$ siècle

Pour citer cet article, to quote this article, para citar este artigo :

Martin Hurcombe « Le Véloce-Sport et l'invention de la culture cycliste », Sur le journalisme, About journalism, Sobre jornalismo

[En ligne, online], Vol 10, n²2 - 2021, 15 décembre december 15 - 15 de dezembro.

URL : https://doi.org/10.25200/SLJ.v10.n2.2021.442 
dans l'évolution des pratiques et cultures sportives à cette époque.

Notre étude se limitera à l'examen de la rubrique « À travers route » et aux articles publiés sous celleci entre 1885 et 1889 , moment pendant lequel le sport commence petit à petit à se démocratiser (Gaboriau, 1991). Sous cette rubrique, on publie les comptes-rendus des périples entrepris d'abord par les membres du VCB et, dès l'automne de 1885, par des membres d'autres clubs vélocipédiques ainsi que par des « indépendants ». Les auteurs de ces récits sont initialement les journalistes du Véloce-Sport et quelques membres $\mathrm{du}$ VCB ainsi que des membres d'autres clubs du sudouest. Pourtant, à la fin de la décennie la rubrique publie les récits d'auteurs du sud-est, de l'est, de la région parisienne, etc. ; elle est donc le reflet de l'importance croissante de la revue bordelaise et de son influence sur le cyclisme au niveau national. Publiée en moyenne une fois toutes les deux ou trois semaines, la rubrique occupe entre une et trois pages sur les seize pages qui composent chaque numéro en moyenne ${ }^{1}$. À la différence de la plupart d'autres rubriques, « À travers route » encourage le lecteur-cycliste de devenir cycliste-écrivain ; elle permet donc de mieux comprendre la contribution de celui-ci à la conceptualisation et à la représentation de la vélocipédie ainsi qu'à une culture cycliste nationale en voie de développement dont on trouve le reflet dans ses récits.

On ne peut pas connaître cette culture sportive naissante sans considérer le lien qui existe entre celleci et son élaboration textuelle dans la presse sportive. Nous soutenons avec Raymond Boyle (2006), Jeffrey Hill (2006) et Philippe Tétart (2015), qu'il ne suffit pas de considérer la presse sportive comme une source ; il faut la traiter en « objet d'histoire » (Tétart, 2015:10) et s'interroger sur ses qualités et pratiques textuelles (tout ce qui se rapporte au discours, à la voix narrative, au style etc.) pour mieux comprendre la manière dont le sport est consommé et pratiqué par ses lecteurs en tant que phénomène culturel et réalité sociale. Notre approche s'inspire également des travaux de Dominique Kalifa, Philippe Régnier, Marie-Ève Thérenty et Alain Vaillant (2011) sur le rôle « civilisant » du journal dans le XIX siècle. Pour ces derniers, il faut tenter de comprendre l'usage fait de la presse par divers groupes sociaux tout en saisissant la manière dont elle « contribue en même temps à façonner, à modeler et à instituer ces différents groupes sociaux [...] » (2011 : 13). Dès lors, le rôle primordial de la presse dans la construction de l'identité et de la culture nationales de l'époque devient évident. De même, étudier une des plus importantes revues cyclistes, et surtout la rubrique à laquelle ses lecteurs ont le plus contribué, nous permet de saisir une culture sportive en voie de développement à la fin du siècle ${ }^{2}$. Cet article tente alors d'apprécier la contribution de la revue cycliste la plus importante de l'époque à ce processus.

Dans un premier temps, nous présenterons au lecteur un résumé de l'histoire et du contenu du Véloce-Sport pour souligner son importance sur la scène nationale, la mission qu'il s'est donnée et les thèmes qui l'ont préoccupé. Nous examinerons ensuite la rubrique « À travers route » entre 1885 et 1889. D'abord, nous analyserons la conceptualisation du temps et de l'espace dans la pratique cycliste (ou vélocipédique pour utiliser le terme de l'époque). Fallait-il, en racontant ses exploits, insister sur la distance et le temps parcourus en « mangeur de route »? Ou devrait-on décrire ce que l'on avait vu, servant ainsi de guide aux futurs touristes ? Ici on trouve deux styles qui co-existent dans les récits des contributeurs : un style télégraphique qui insiste sur la vitesse et la distance parcourue et un style plus lyrique qui met en valeur l'intérêt des paysages et villes traversés. Ces deux styles sont le reflet d'une division au sein du sport entre ceux qui accordent une priorité aux courses et ceux qui prônent le cyclo-tourisme, division qui finira par un schisme au sein de l'UVF en 1890 et qui mènera à la création d'une organisation rivale : le Touring Club de France.

Dans un deuxième temps, nous nous pencherons sur la mise en valeur dans ses récits de la sociabilité masculine qui se construit autour des activités des sociétés vélocipédiques d'où émergera l'image du véloceman tel qu'il est conçu par les pratiquants du sport dans les années 1880 . Terme utilisé jusque-là pour désigner tout adepte de ce qu'on appelle pour l'instant la vélocipédie, le véloceman prend de l'envergure dans les pages du Véloce-Sport. C'est autour de cette vision quelque peu idéalisée du véloceman que la vélocipédie est conçue comme une véritable pratique sportive et culturelle à la fin du XIX ${ }^{\mathrm{e}}$ siècle. Nous verrons ainsi comment le fait d'écrire sur le cyclisme sert à fonder une culture cycliste à travers une conceptualisation dominante du temps et de l'espace et son personnage principal.

\section{LA MONTÉE DU VÉLOCE-SPORT}

Le premier numéro du Véloce-Sport paraît le 5 mars 1885 . Vendu à 25 centimes (12 francs pour un abonnement annuel), il est le reflet de l'essor de la revue spécialisée de la fin du XIX ${ }^{\mathrm{e}}$ siècle et de la diversification des sujets et des lieux de publication sous la Troisième République (Geslot et Hage, 2002 : 35-36). Tétart constate par exemple que, entre 1890 et 1910 , 561 périodiques sportifs voient le jour en France, dont 369 en province (2015: 11). Le périodique bordelais profite également d'un appétit grandissant pour les hebdomadaires (Loué, 2002 : 62) surtout lorsque la 
pratique de la vélocipédie se démocratise encore plus rapidement dans les années 1890 . Dès 1887 il se vend à Lyon, Marseille, Paris, Bruxelles et Turin, ainsi que dans toutes les agences de journaux suisses. En 1890 on peut l'acheter un peu partout en France et chez quatre librairies à Londres. Cette même année la rédaction est partagée entre Bordeaux et Paris où, à partir de 1893, Le Véloce-Sport est co-locataire d'une salle télégraphique avec le quotidien Le Vélo. Ce dernier est lancé par Pierre Giffard et Paul Rousseau, un des membres du comité de rédaction du VéloceSport ${ }^{3}$. L'essor du cyclisme de la première moitié des années 1890 permet une réduction continue du prix du Véloce-Sport qui descend à 10 centimes le numéro en 1892 à un moment où il est vendu sur tout le territoire français ainsi qu'aux Pays Bas et en Espagne. Le succès immédiat du Véloce-Sport est également évident dans sa capacité d'absorber son plus grand rival en octobre 1886 : Le Véloceman de Montpellier. Ayant fusionné avec La Bicyclette en 1896, il publia son dernier numéro en novembre 1897.

L'importance nationale du Véloce-Sport dans l'élaboration de la culture cycliste, ce qui justifie d'ailleurs notre choix parmi les diverses revues cyclistes de l'époque, réside également dans son rapport avec l'UVF. Dans son premier numéro, son rédacteur-enchef, Fernand de Laclos (nom de plume de Fernand Ladevèze), prête serment d'allégeance à l'UVF tout en déclarant une ligne éditoriale indépendante qui permet au Véloce-Sport de critiquer «les vices » de l'organisation nationale et « les erreurs dans lesquelles peuvent tomber ceux qui sont à sa tête ». Comme le constate Hugh Dauncey, l'UVF eut du mal à imposer ses tendances centralisatrices sur les organisations et clubs provinciaux (2012: 46). Le fait que Le VéloceSport en devient l'organe officiel en 1888 est le reflet d'une victoire au moins partielle d'une conceptualisation du sport articulée dans les pages du périodique bordelais.

Pourtant, l'élaboration d'une culture cycliste n'est pas l'œuvre d'une élite journalistique basée initialement à Bordeaux qui finit par s'imposer sur la nation. Les premiers contributeurs au Véloce-Sport sont surtout membres du VCB, mais les ambitions nationales du périodique et son ouverture d'esprit vers un lectorat plus élargi sont évidents dans son programme du premier numéro où Laclos conclut par une invitation : « Nos colonnes sont ouvertes à tous les vélocipédistes, à tous ceux qui ont à cœur de contribuer au progrès du sport qui nous est cher » (Le Véloce-Sport, le 5 mars 1885 : 1). Le Véloce-Sport est une entreprise collective où la distinction entre journaliste et lecteur reste floue, une frontière que ce dernier est invité à traverser, surtout sous la rubrique « À travers route » dont l'existence même dépend du cycliste-écrivain.
Il faut constater que Le Véloce-Sport est lancé sur le marché de la presse cycliste à un moment où le sport est caractérisé par une diversité de modèles. Ce n'est qu'au début des années 1890 que la célèbre safety bike commencera à s'imposer comme le choix préféré des cyclistes européens et américains. Entre 1885 et 1890 , deux autres modèles font concurrence à la bicyclette telle qu'on la connaît de nos jours : le tricycle et le grand-bi. Les trois modèles co-existent à partir de 1885 suite au lancement de la première «Rover safety bike » en Grande Bretagne (Herlihy, 2004 : 227-40). Pendant le moment qui nous intéresse, le cyclisme français est partagé entre différentes conceptions du sport que l'on nomme pour l'instant la vélocipédie ainsi que par différentes conceptions de son objet ${ }^{4}$.

Plusieurs questions sous-tendent la structure et le contenu du Véloce-Sport dans les années 1880. D’abord, à quoi pourrait servir cette nouvelle «machine »? Comment l'utiliser ? Quelles en sont limites ? Jusqu'à où peut-on aller et à quelle vitesse ? Et qui a le droit de s'en servir ? Une partie de l'hebdomadaire est consacrée aux affaires de l'UVF, surtout à partir de 1888, et les débats qui divisent les cyclistes, par exemple, en partisans des courses ou du cyclo-tourisme. Le VéloceSport consacre une bonne partie de chaque numéro à la campagne de promouvoir la valeur du cyclisme pour l'armée française. Selon celle-ci, le cyclisme pouvait non seulement contribuer à la réhabilitation physique des Français suivant la défaite humiliante lors de la guerre franco-prussienne, mais le vélocipède pouvait devenir un outil de l'armée moderne, permettant à l'infanterie de se déplacer plus rapidement, par exemple (Le Véloce-Sport, $1^{\mathrm{er}}$ octobre $\left.1885: 458\right)^{5}$. La rubrique « L'Hygiène du vélocipédiste » offre les conseils du Dr. Tissié qui fait l'éloge des bienfaits du sport pour la santé tout en avertissant le lecteur contre la fatigue et le « surmènement $»^{6}$. Les courses cyclistes ( «Echos de la piste ») et les activités des clubs vélocipédiques qui comprennent surtout des sorties officielles et nonofficielles (en petits groupes ou en masse) et, plus rarement, des tentatives de «recordmen » sont aussi en évidence dans tous les numéros. Sur le plan pratique, un article par numéro est consacré aux nouveaux produits et développements industriels et chaque numéro contient une liste de « Maisons recommandées » où manger ou se loger au cours d'une longue excursion. Les publicités (pour vélos, accessoires, costumes, boissons fortifiantes) occupent, en règle générale, une place très importante dans l'hebdomadaire bordelais (d'habitude 25\%). Mais c'est la rubrique « À travers route » qui nous intéresse surtout car c'est sous elle que les lecteurs du Véloce-Sport prennent la parole et sous elle qu'une certaine manière de raconter le voyage à vélo commence à s'imposer ainsi qu'une certaine image du véloceman esquissée un peu partout sous d'autres rubriques. 


\section{MANGER OU SAVOURER LA ROUTE ?}

Les premiers articles à paraître sous cette rubrique sont signés par Sun, membre du VCB, qui raconte son voyage à tricycle en réponse à un appel de Nestor dans un périodique rival, Le Sport Vélocipédique. Ceci encourage les « recordmen » à raconter leurs déplacements afin de renseigner le lecteur sur l'état des chemins et la meilleure route à emprunter tout en l'épatant de la distance et du temps parcourus ( $L e$ Véloce-Sport, le 16 juillet 1885 : 281). L'écrivain-cycliste envisagé par Nestor et incarné d'abord par Sun est un auteur de guide, mais également un peu journaliste rapportant lui-même pour ses lecteurs ses propres réussites vélocipédiques. Très vite, d'autres membres $\mathrm{du}$ VCB prennent la plume pour raconter leurs aventures. À partir de mai 1886, on trouve de plus en plus de récits d'excursions entreprises par d'autres sociétés vélocipédiques (notamment, celles de Grenoble, Narbonne et Angers). En 1887, la gamme géographique des contributeurs et des destinations, qui s'étend désormais sur la majorité du territoire national, est le reflet du succès grandissant du périodique bordelais.

Si l'invitation de Nestor contenait l'ébauche d'un contenu, toujours est-il qu'il fallait trouver un style et un langage aptes à capturer l'expérience. La mise en valeur de certains thèmes, la façon dont le temps et l'espace sont évoqués, bref, la façon de raconter le cyclisme restent pour l'instant le choix du cyclisteécrivain. Raconter ses aventures, c'était aussi imposer sa propre vision de la vélocipédie, répondre aux questions qui sous-tendaient les représentations du sport naissant sous d'autres rubriques du Véloce-Sport, et accepter ou résister à certains courants ou croyances qui y circulaient.

Dès les premiers comptes-rendus des membres du VCB, pourtant, on constate l'usage d'un style qu'on peut qualifier de télégraphique qui sera adopté par bien d'autres contributeurs au cours des années 1880 . « Voyages de deux membres du V.C. bordelais », écrit par le « recordman » et père fondateur du VCB Jiel (pseudonyme de Joseph Marie François Laval), commence sur un ton lyrique ( «lorsque le temps me procure ses faveurs [.... ») qu'il abandonne très vite. Dès lors, le récit se réduit à un itinéraire qui détaille le temps et les villes parcourus ainsi que les repas et l'alcool consommés sur 325 kilomètres : «Après un quart d'heure d'arrêt nous repartons, et atteignons Saint-Médard (59 kil.) à 5 h45. Trois minutes d'arrêt, le temps d'absorber un Picon [...] » (Le Véloce-Sport, le 30 avril 1885 : 105). Le récit de Jiel sert de démonstration des capacités d'endurance et de vitesse des deux cyclistes et offre la preuve au lecteur que les deux vélocipédistes sont « encore dignes d'être de la section des mangeurs de route $[\ldots] »(106)$, mais refuse toute description détail- lée du paysage. D’ailleurs, on trouve le même langage dénudé dans « De Beregrac à Saint-Yrieix » de E. Laururie du Sport Vélocipédique de Bergerac. Si l'objectif du périple est de visiter plusieurs châteaux, chaque visite ne constitue qu'un « Arrêt de quelques minutes [...] ». Pour encore réduire la longueur du récit, l'auteur abandonne les pronoms personnels : «Arrivons à Jumillhac-le Grand (14 kil.) par une pente douce de 5 kilomètres. Admiré le château » (Le Véloce-Sport, 28 avril $1887: 254$ ).

Si Sir Ericon, l'auteur de «De Bordeaux à Toulouse et retour », adopte le style télégraphique qui est devenu la norme stylistique des récits des tentatives de record sur distance, indiquant les villes par lesquelles les deux vélocipédistes sont passées, détaillant carrefours, collines etc., il décrit en détail la condition des routes pour offrir au lecteur un texte hybride au croisement du récit sportif, de la carte topographique et du guide touristique : «Route médiocre jusqu'à SaintBazeille, très bonne jusqu'à Marmande où nous arrivons à huit heures quarante et où nous faisons notre premier déjeuner à l'hôtel de la Providence. (Très recommandé aux touristes qui ne veulent pas rentrer en ville.) Il est situé au croisement de la route nationale no 133 (83k 80o) » (Le Véloce-Sport, le 12 juillet 1888 : 430). L'auteur répond donc indirectement aux tentatives de l'UVF de réunir deux tendances au sein du cyclisme français (Dauncey, 2012 : 47) : d'une part, les « recordmen » et vélocemen voués aux records et aux courses ; de l'autre part, les cyclo-touristes pour qui le plus important était « la ballade [sic] à sa guise, l'indépendance, toutes choses qui s'accommodent fort peu des règlements de temps et de distance » $(R e$ vue mensuelle du Touring Club de France, février 1891 : 39). Sous la rubrique « À travers route » ces deux tendances peuvent cohabiter dans le même texte ; ainsi un certain « E.L. » (peut-être le même E. Laururie cité ci-dessus) adopte-il le style télégraphique dans son compte-rendu d'une excursion qu'il interrompt brièvement pour décrire la perspective d'une colline, par exemple, tout comme lui et ses compagnons s'arrêtent pour visiter l'église abbatiale de Cadouin dans le but d'y voir le suaire qui est censé avoir enveloppé le Christ (Le Véloce-Sport, 30 avril 1885 : 106).

Les lecteurs du Véloce-Sport, tout comme les membres du VCB (Dauncey, 2012 : 26), sont partagés dans les années 1880 entre « recordmen » et touristes. Les récits de « À travers route » oscillent donc entre un lyrisme qui s'associent aux valeurs de ces derniers et un style télégraphique censé être le reflet de la vitesse de ces premiers. Pourtant, le lyrisme de ces récits est souvent miné par un ton ironisant adopté volontiers par ces auteurs comme s'ils se moquaient des prétentions littéraires qui caractérisent un certain journalisme de qualité. Ainsi se protègent-ils contre toute critique ; comme l'affirme Myriam Boucharenc, 
journalisme et littérature s'entrecroisent sous la Troisième République sans que ce premier se débarrasse d'un fort sens d'infériorité vis-à-vis de cette dernière qui, elle, n'hésite pas à le remettre à sa place (2004 : 1926). Sun adopte ainsi une tactique d'auto-défense dans son récit « La Fontaine Rollet » quand il évoque une journée ensoleillée sur un ton ironique : «J'avance toujours, et maintenant Phébus inonde de lumière la campagne riante ». Pourtant, il s'oublie quelques instants plus tard. La chaleur l'empêche de rouler vite et il se perd par la suite dans la contemplation des couleurs et des sons qui l'entourent : « le bruissement des feuilles se mêle au murmure de l'onde ; on entend dans le lointain la voix des laboureurs [...], le tintement des cloches du troupeau voisin allant au pâturage ; tout cela forme une musique mélodieuse, avec des sons variant à l'infini ». Enveloppé par la nature, il se perd dans le moment. Il ne mange plus la route comme son co-équipier Jiel ; il le savoure et consacre son récit à « faire connaître aux vélocipédistes cet éden bordelais » (Le Véloce-Sport, le 20 août 1885 : 357), le vouant ainsi au tourisme.

Par ailleurs le regard de Sun et d'autres contributeurs à la rubrique « À travers route » est attiré souvent sur les sites historiques qui deviennent pour certains l'objectif principal du périple. Dans la plupart des cas, pourtant, les descriptions de celles-ci sont toutà-fait banales et dépourvues de tout intérêt littéraire ou historique. Dans « Véloce-Club grenoblois dans les Alpes », par exemple, Novice n'évoque le paysage qui l'entoure ( « cette splendide est si pittoresque route des gorges de la Bourne, qui se développe dans un paysage grandiose par son horreur, la sauvagerie de ses sites et le nombre de ses gouffres ») que de façon sommaire (Le Véloce-Sport, le 13 mai 1886 : 224). En effet, le mot pittoresque sert de poncif descriptif dans bien de récits, un peu comme le fera quelques décennies plus tard l'icône « point de vue » sur les cartes routières, pour signaler au lecteur qu'il y a bien quelque chose à contempler. Mais, vers la fin de la décennie, la description fait place à une simple liste de sites d'un intérêt éventuel. L'auteur de « De Decize (Nièvre) à Cannes (670 kilo) », un certain C. Nicard, négociant en bois de la Haute-Savoie, refuse carrément le rôle de guide touristique pour insister plutôt sur son passage à travers le paysage, son combat avec le Mistral et son triomphe sur celui-ci :

$\ll[\mathrm{J}] \mathrm{e}$ vous dirai que je pourrai bien me servir du Guide Joanne et vous faire le récit de telle ou telle pierre, de telle ou telle église du XII ${ }^{\mathrm{e}}$ ou du XIII ${ }^{\mathrm{e}}$ siècle, etc., etc., mais je n'ai pas le temps, et puis je préfère [...] négliger toutes les citations archéologiques qui intéresseraient fort peu vos lecteurs, lesquels, j'en suis sûr, préféreront savoir comment j'ai pu, en bon bourgeois, en vrai bicycliste, atteindre le but de mon voyage. Là-dessus, je poursuis, laissant à d'autres, plus érudits que moi, le soin de vous faire ces longues descriptions pleines de science, mais arides et sèches comme les dunes d'un désert. » (Le Véloce-Sport, le 19 janvier $1888:$ 1280)

L’intérêt des lieux traversés se réduit uniquement alors à son rapport à la vitesse et à la difficulté du périple. En ceci, Nicard anticipe les conseils du comité de rédaction aux contributeurs qui déclare le 5 avril 1888 que les récits de sorties doivent faire preuve d' « un développement proportionné à l'importance de l'excursion qu'ils auront à raconter ». Et de conclure : « Il est bon d'être concis et d'éviter les détails qui, forcément communs à toutes les promenades, en deviennent banales » même si le comité de rédaction ne ferme pas définitivement la porte à « des narrations vraiment intéressantes, amusantes et instructives [...] » (141). Ces consignes éditoriales constituent une tentative d'imposer un style aux récits et en même temps une manière de concevoir les villes et les paysages traversés à bicyclette, un style qui favorise la brévité et la vitesse. Pourtant, si les excursions racontées dans les pages du Véloce-Sport nous apprennent qu'il faut plutôt manger que savourer la route, elles servent un deuxième objectif tout aussi important à la construction d'une culture cycliste : promouvoir à travers le récit une sociabilité sportive exclusivement masculine et surtout bourgeoise résumé dans le personnage du véloceman.

\section{« NE PAS PERDRE UNE MINUTE »}

La sortie des sociétés ou clubs vélocipédiques avait plusieurs buts. D'abord, il s'agissait d'entraînement en dehors des courses organisées sur « pistes ». (Dans la majorité des cas, et avant la construction en masse des vélodromes à partir des années 1890, celles-ci consistaient en des tours des places principales, par exemple la Place des Quincones à Bordeaux, pour les courses organisées par le $\mathrm{VCB})^{7}$. C'était également un moyen de s'entraîner pour les tentatives de record entreprises entre deux villes (parfois en aller-retour et presque toujours sur plusieurs centaines de kilomètres). Pourtant les récits de ces sorties mettent en valeur surtout leur fonction de sociabilité masculine et bourgeoise. Comme l'explique Varda Burstyn, le sport à la fin du $\mathrm{XIX}^{\mathrm{e}}$ siècle en Europe et dans le monde anglophone est un domaine réservé presque exclusivement aux hommes ; il sert donc à créer et à conforter les liens de sociabilité et la domination masculines (1999 : 5153). Les récits de sorties de clubs racontés dans $L e$ Véloce-Sport sont le reflet de cette exclusivité masculine ${ }^{8}$, mais, ce qui est plus important, ils contribuent à la construction de l'identité cycliste en créant le personnage du véloceman. Celui-ci prend sa réalité dans l'acte de raconter ses journées passées en selle et ses rapports avec d'autres hommes de la même classe sociale $^{9}$. 
Les sorties non-officielles des membres de sociétés vélocipédiques, par exemple, semblent être surtout motivées par le désir de passer du temps en bonne compagnie. Le récit sans titre de Félix Roger du $1^{\text {er }}$ octobre 1885 en offre un bon exemple. Le but de sa sortie avec son ami Georges, avec qui il partage un logement, est de «tuer le temps ». Le véloceman idéal dans ces récits est un bon bourgeois qui dispose du temps, et donc qui le possède en quelque sorte. Il réveille Georges ainsi : « Good morning dear ! [...] Il s'agit, mon cher, de ne pas perdre une minute. 'Time is money,' comme disent nos voisins d'outre-Manche ». L'usage de l'anglais (qu'on remarque aussi dans les pseudonymes de bien des contributeurs au VéloceSport) signale un désir d'imiter un certain esprit et style associés à l'aristocratie anglaise, même s'ils sont utilisés de façon ironique. Roger est donc typique d'une bourgeoisie française « incapable de créer par elle-même une mode » à qui il faut « des modèles » parmi lesquels on trouve des « sportmen anglais [...] » (Gaboriau, 1991:21).

Le récit de Roger abandonne très vite toute tentative de décrire les «pittoresques beautés » de la route qui mène à Thiers (Puy-de-Dôme) car elle est trop dangereuse et l'auteur n'a que « l'œil ouvert aux cailloux » (Le Véloce-Sport, $1^{\mathrm{er}}$ octobre $\left.1885: 456\right)$. Il détaille plutôt l'alcool consommé (Picon, cognac, liqueurs et bocks), ses effets sur les deux vélocipédistes et leur capacité diminuante de maîtriser leurs vélocipèdes. Le but de Roger est d'avertir le lecteur tout en l'amusant, mais il insiste surtout sur la convivialité et le plaisir de faire du vélo en bonne compagnie masculine.

La convivialité est également le thème dominant chez les récits de sorties officielles des clubs. Cellesci prennent parfois la forme d'une rencontre sociable avec un club voisin où toute rivalité sportive est suspendue. Ainsi un numéro de janvier 1888 contient-il le récit d'une promenade réunissant le C.V. de Sayon et le club de Tonnay ainsi qu'un deuxième qui raconte la rencontre entre le VCB et le V.C. Libournais ; « cette charmante fête de famille » comprend un déjeuner copieux précédé d'apéritifs («byrrh, picon, madère, etc. ») (Le Véloce-Sport, le 19 janvier 1888 : 1278). L'excursion de la SVM entreprise en août 1887 (en toute probabilité la Société Vélocipédique Métropolitaine), raconté par C.M., est structurée autour d'une série de rencontres et de banquets avec d'autres sociétés vélocipédiques « où nous sommes reçus de la façon la plus cordiale » (« Excursion de la SVM », Le Véloce-Sport, $1^{\mathrm{er}}$ septembre $\left.1887: 784\right)$. En préparant leur excursion, la SVM avaient prévenu toutes les sociétés vélocipédiques des villes traversées, mais il faut constater qu'au moins trois sociétés n'ont pas répondu à leur appel. Toujours est-il que, selon Novice du Véloce-Club Grenoblois, la « caravane [de deux sociétés réunies] au bruit des chants joyeux et des gaudrioles contées » sert « [et] le bien de la vélocipédie et la recherche de la bonne vieille gaieté française » (Le Véloce-Sport, 13 mai $1886: 224$ ).

Qu'il s'agisse de rencontres entre clubs ou entre individus du même club, l'important est de se montrer gentleman (et le mot anglais est employé régulièrement dans les pages du Véloce-Sport). Se conduire en gentleman c'est rester respectueux à tout moment envers tout autre véloceman pourvu que ce dernier soit de la classe méritante ${ }^{10}$. Les vélocipédistes de la petite bourgeoisie constituent un "voisinage compromettant $[. .]$.$» qu'il faut éviter sur les routes selon$ Sun (Le Véloce-Sport, le 3 septembre $1885: 387$ ), alors que reconnaître un membre ami du même club offre l'occasion de se montrer véritable gentleman. Sun et ses compagnons essaient de fausser compagnie à un cycliste de la petite bourgeoisie, par exemple, qui essaie de rouler avec eux. Lorsqu'il n'abandonne pas la poursuite, ces gentlemen du VCB semblent décider de recourir à la violence et l'attend sur un pont espérant qu'il passera par-là peut-être dans l'espoir de lui régler son compte (Le Véloce-Sport, le 3 septembre 1885 : 388). Par voie de contraste, lorsque Sun avait rencontré Lickson, son compagnon de route pour la journée qui s'étendait devant eux, sur ce même «magnifique pont de Bordeaux [...] » deux mois plus tôt, les deux vélocipédistes s'étaient lancés dans un échange théâtral qui est l'expression même de l'amabilité cycliste et de la conduite d'inspiration aristocratique qui la soustend. Raconter cette rencontre est aussi une façon de montrer au lecteur le rôle qu'il faut assumer pour paraître véritable véloceman :

\section{Tiens ! Sun !}

C'est vous, Lickson. Mes compliments, mon cher ; l'exactitude est une des vertus du véloceman. (Le Véloce-Sport, le 16 juillet 1885 : 281)

Reconnaître un véloceman, plutôt qu'un parvenu à vélocipède d'occasion, est facile grâce au costume porté par les membres des sociétés vélocipédiques et les cyclistes plus aisés. Adosser son « costume de véloceman [...] » devient pour Joseph Vigne une façon d'assumer le personnage du cycliste moderne qui se réveille pour déjeuner rapidement à quatre heures du matin avant de se mettre en selle (Le Véloce-Sport, le 22 octobre $1885: 500$ ). Mais le costume devient uniforme quand il s'agit de couleurs de club et d'une sortie officielle. Son port est obligatoire car il fait partie intégrante du caractère quasi-militaire de ces activités. La sortie officielle du VCB raconté dans le numéro du 19 janvier 1888 met ainsi en lumière son organisation qui imite celle d'une armée en manœuvres. Le président Rousset mène le train tandis que « J. Laval (triPhénix), capitaine de route, [ferme] la marche pour ramasser les blessés et exciter les retardataires [...] » et 
« M. Lefaux (tri-Rudge) [part] en éclaireur [...]» (Le Véloce-Sport : 1278). Le rôle du capitaine est de relever « les esprits chancelants [...] » (Le Véloce-Sport, le 8 mars $1888:$ :79) et de rappeler à ceux qui parlent de rentrer en prenant le train « que cela était indigne des vélocipédistes, et qu'il fallait faire contre mauvaise fortune bon cœur [...] et qu'on n'entendra parler du chemin de fer que pour entrer en lutte avec lui [...] » (Le Véloce-Sport, le 8 mars 1888 : 78). Écrire la sortie officielle sert à renforcer les valeurs clefs associées au véloceman ainsi qu'à son club ; à savoir, le flegme, la volonté, l'endurance et l'esprit de corps qui émane de l'identité d'équipe.

Le rapprochement effectué ici entre les sorties officielles des clubs et les manœuvres d'une armée en marche rappelle au lecteur les possibilités militaires du vélocipède dont il est question ailleurs dans Le VéloceSport. Comme le passage des troupes en manœuvres, « La file des vélocipédistes produit sensation sur la masse des promeneurs, qui [...] envahit la rue Nationale [à Tours] » (Le Véloce-Sport, le 5 avril $1888: 142$ ). La traversée de la campagne et des villes est ressentie comme une espèce de conquête de l'espace et du temps par les auteurs de ces récits. Si les membres du Véloce-Club du Gard sont poussés à voir « cette ancienne ville de Baux [dont] il reste encore de si curieux vestiges $[. .$.$] . , c'est pour en prendre des photo-$ graphies qui finiront par « orner la salle du V.C.G. » (Le Véloce-Sport, le 10 mai 1888 : 246). D’autres préfèrent le dessin pour enregistrer leur visite ou même pour laisser leur empreinte en inscrivant les initiales ou l'écusson de leur club sur un rocher (Le VéloceSport, 27 août 1885 : 372). L'imaginaire conquérant de certains contributeurs se traduit même par un ton, un langage et une vision quasi coloniaux lorsqu'ils racontent la découverte du territoire national. Le récit d'une excursion de la S.V. Métropolitaine en Oise (par un certain Cow-Boy) traite les habitants de Beauvais en indigènes aux « cervelles rétives » (Le Véloce-Sport, le 26 juillet $1888: 473$ ) puisqu'ils ne savent pas distinguer entre tricycles, bicyclettes et grand-bis. Par ailleurs, E.L. traite les habitants de Chavagnac (qui est « un trou ») en « sauvages » (Le Véloce-Sport, le 30 avril $1885: 107)$. Le récit de la sortie officielle est le reflet donc non seulement de la découverte du territoire national et de l'apprentissage des régions encouragés par la Troisième République pour fonder l'unité nationale, mais aussi de sa politique coloniale expansionniste et un certain enthousiasme pour ceci promu dans une propagande en faveur du colonialisme. Ces deux mouvements sont bien sûr réunis dans le personnage de Jules Ferry, Ministre de l'Education Nationale et ensuite Ministres des Affaires Etrangères, entre 1879 et 1885 . Il ne faut pas oublier non plus qu'en 1888 , l'année où paraissent un nombre des récits étudiés cidessus, le boulangisme est en plein essor et est accompagné d'un vif culte de la vie militaire. Pour Jacques
Augendre, il ne s'agit de rien de moins qu'une époque « où le cyclisme devient une discipline aristocratique et une discipline... militaire » (1998: 215). Même si les adeptes de la vélocipédie sont surtout des membres de la bourgeoisie dans les années 1880 (Gaboriau, 1991 : 19), on peut parler d'une militarisation plus générale des esprits et surtout de la masculinité française à cette époque qui envahit le sport mais qui en est nourri en même temps (Burstyn, 1999). Le pseudo-militarisme de la sortie officielle telle qu'elle est racontée sous la rubrique « À travers route » met en valeur la conquête de l'espace et du temps plutôt que l'intérêt historique du terroir traversé. Ce genre de récit évite surtout la description des lieux et des paysages qui deviendra par la suite un des objets de la littérature cyclo-touristique des années $1890^{11}$. Et, comme pour confirmer ce fait, le comité de rédaction intervient encore une fois en juillet 1888 pour signaler que « Notre journal n'est pas une feuille littéraire, les courses et excursions doivent être présentées sous une forme très courte ; quand un lecteur voit un article long, il en remet la lecture à une autre fois et on sait ce que cela veut dire » (Le VéloceSport, le 26 juillet $1888: 477$ ).

\section{CONCLUSION}

Écrire la sortie d'un club vélocipédique devient un moyen d'affirmer et de concrétiser son existence aux yeux du lectorat national (voire, international) du Véloce-Sport. C'est aussi une façon de signaler son adhésion à l'UVF, à ses mœurs et à ses valeurs. Pour Bramwells, capitaine du Vélo-Sport Parisien, « capturer » la première excursion de son club dans les pages de l'hebdomadaire de l'UVF, est un moyen d'insister sur « la franche gaîté, la cordialité et le bon accord qui [règnent] parmi nous. C'est un bon augure pour l'avenir » (Le Véloce-Sport, le 10 mai 1888 : 245). Écrire le cyclisme est ainsi une façon d'afficher son appartenance à une culture cycliste émergeante en France. C'est aussi contribuer à sa construction et à son élaboration.

Pourtant, et comme nous l'avons déjà constaté, la façon de raconter l'excursion à vélocipède devient un choix entre l'expression de deux expériences que certains à la fin des années 188 o veulent diamétralement opposées. Même si le comité de rédaction affirme que « Notre sport est assez grand pour tous ! » en mars 1888, elle insiste dans le même article sur le fait que la fonction originelle (et donc fondamentale) de l'UVF est l'organisation du sport compétitif (Le Véloce-Sport, le $1^{\mathrm{er}}$ mars $1888: 47$ ). Ce même éditorial fait allusion au schisme grandissant au sein de l'UVF entre ceux qui suivent la ligne du Véloce-Sport et ceux qui considèrent le tourisme comme l'avenir du cyclisme à un moment où les courses et les records attirent de moins en moins l'attention du grand public. Ce schisme mène directe- 
ment à la création du Touring Club de France en 1890 qui, dans ses premières années, s'intéressent surtout au cyclisme et à la marche à pied avant de virer vers l'automobilisme.

Par voie de contraste, Le Véloce-Sport se lance dans la promotion et l'organisation de la première grande course sur route en mai 1891 : Bordeaux-Paris, course qui sera dominée par des coureurs anglais. Pour remonter le moral des Français, et pour confirmer la domination de la safety bike ainsi que son utilité générale, Pierre Giffard du Petit Journal lance la première ParisBrest-Paris en septembre du même an. La participation étant réservée aux citoyens français, elle finit par une lutte entre Charles Terront (de l'équipe Michelin) et Jiel-Laval (ancien contributeur à la rubrique « À travers route » et membre de l'équipe Dunlop). La victoire de Terront sur l'ancien président du VCB marque encore une évolution dans la course sur route. A partir de ce moment, et surtout avec l'avènement de l'automobile, la bourgeoisie se désintéresse du cyclisme, cédant la place aux champions d'origines plus humbles, comme Terront (Dauncey 2012 : 25). Deuxième évolution : l'avènement d'une presse sportive nationale qui voit dans les courses cyclistes et autres événements sportifs un moyen d'augmenter ses ventes. Giffard, par exemple, quittera Le Petit Journal pour fonder Le Vélo en 1892. Celui-ci entrera en conflit avec L'Auto-Vélo d'Henri Desgrange, père fondateur du Tour de France en 1903, pour lequel la course cycliste annuelle devient le garant d'un revenu sûr.
Les rédacteurs du Véloce-Sport n'auront pas le loisir de discuter cette grande rivalité ; leur hebdomadaire disparaît en 1897 « cédant à son tour à la loi du progrès [qui dicte que] la place des informations sportives n'est plus dans les publications hebdomadaires » (Le Véloce-Sport, le 11 novembre 1897 : 9). Mais il s'agit tout autant de la fin d'une certaine image du cyclisme en tant que sport promue par Le Véloce-Sport. Celle-ci voulait associer le sport à un amateurisme absolu et à une aisance bourgeoise dont le véloceman et la société vélocipédique étaient devenus les symboles. La montée du véloceman des années 1880 est de courte durée ; le cyclisme se démocratise de plus en plus rapidement à partir des années 1890 et les vélocemen cèderont leur place au début du $2 \mathrm{O}^{\mathrm{e}}$ siècle aux « ouvriers de la pédale » de Desgrange. Toujours est-il que, au cours de ses douze années d'existence, Le Véloce-Sport établit les bases de la culture cycliste en France, bases sur lesquelles les Giffard et Desgrange surent construire le sport qui allait dominer la vie sportive de la France dans la première moitié du $\mathrm{XX}^{\mathrm{e}}$ siècle. L'analyse du Véloce-Sport montre comment la presse sportive représente un élément constituant de l'émergence d'une culture sportive en France à la fin du XIX ${ }^{\mathrm{e}}$ et au début du $\mathrm{XX}^{\mathrm{e}}$ siècle. C'est en scrutant cette presse d'un œil plus attentif et en s'interrogeant sur ses qualités et pratiques textuelles qu'on se rend compte pleinement de cette contribution.

Proposé le 9 octobre 2020

Accepté le 9 juillet 2021

\section{Notes}

\footnotetext{
1. La rubrique paraît pour la première fois en juillet 1885. En 1885 , elle paraît 13 fois ; en 1886, 16 fois ; en 1887, 22 fois ; en 1888, 28 fois ; en 1889, 22 fois. En 1887, 46\% des récits sont sous-titrés «Sortie officielle » d'un club quelconque (36\% en 1888).

2. Il faut insister que, dans les années 1880 , le journalisme sportif est surtout une affaire d'amateurs et que, dans le monde du cyclisme, tout journaliste est un sportif et tout sportif est invité à tenter sa main en tant que journaliste.

3. Rousseau devient responsable de la rubrique sportive du Temps en 1904 avant de travailler pour L'Excelsior en 1910 (Tétart, 2015 : 13).

4. Voir « Vélo, bicyclette : histoire des mots » (Vallet, 1998) pour une histoire de l'évolution des termes associés au cyclisme.

5. Pour un résumé des débats sur le cyclisme militaire, voir Christopher Thompson (2006:27-30).

Sur le concept de « surmènement » à la fin du siècle, voir Robert Nye (1984 : 324) et Varda Burstyn sur son rapport au sport (1999 78-80 ; 99-100).

Pour une histoire du vélodrome en France dans le contexte du sport-spectacle, voir Robert W. Lewis (2016).

8. Nous avons trouvé une seule exception : C.M. raconte dans « Excursion de la S.V.M. » une rencontre avec « la famille Leclerc, d'Evreux [.... » qui comprend deux filles à tandem, Jeanne (« une excellente vélocewoman ») et Cécile (Le Véloce-Sport, le $1^{\text {er }}$ septembre $1887: 783$ ).
}

9. On trouve quand même la présence de cyclistes qui ne ressortent pas de la grande bourgeoisie dans les pages du Véloce-Sport, mais ils restent des personnages en marge du récit. La présence de Georges Juzan, mécanicien, constructeur et inventeur qui tenait un atelier à Bordeaux, et qui a donc joué un rôle primordial dans le développement du sport dans le sud-ouest, ainsi que celle d'un de ses ouvriers, sont à peine évoquées dans un récit de Sun dont l'auteur et Lickson restent les personnages principaux et les seuls qui ont droit à la parole (Le Véloce-Sport, le 16 juillet 1885 : 281-82).

10. Comme le constate Dauncey, le développement des sports à Bordeaux est lié à la présence d'une population britannique et exprime une volonté d'émuler son développement outre-manche (2012: 21)

11. C'est surtout la Revue mensuelle du Touring Club de France qui en publiera dès 1891 les meilleurs exemples, mais un bon nombre de livres qui racontent le long périples de leurs auteurs à travers la France et l'Europe sont également publiés au cours des années 1890 ; pour ne citer que deux exemples : Dieppe, Middelburg, Bruxelles. Huit jours à bicyclette d'Oswald Hefty (Paris : Librarire Sportive, 1894) et La France en bicyclette. Étapes d'un touriste, de Paris à Grenoble et à Marseille de Jean Bertot (Paris : LibrairiesImprimeries réunies, 1894). Les deux sont également membres du TCF. 


\section{BIBLIOGRAPHIE}

Augendre, J. (1998). « La loi des fédérations », Les cahiers de médiologie, 5, pp. 213-19.

Boucharenc, M. (2004). L'Écrivain-reporter au cour des années trente. Lille, Presses Universitaires du Septentrion.

Boyle, R. (2006). Sports Journalism : Context and Issues. London, Thousand Oaks et New Delhi, Sage Publications.

Burstyn, V. (1999). The Rites of Men : Manhood, Politics and the Culture of Sport. Toronto, Toronoto University Press.

Dauncey, H. (2012). French Cycling : A Social and Cultural History. Liverpool, Liverpool University Press.

Gaboriau, P. (1991). « Les trois âges du vélo en France », Vingtième Siècle, revue d'histoire, 29, pp. 17-34.

Geslot, J-C et Hage, J. (2002). « Recenser les revues » in Pluet-Despatin, J. Leymaire, M. et Mollier, J-Y, La Belle Époque des revues. Condé-sur-Noireau, Éditions de l'IMEC, pp. 29-42.

Herlihy, D. (2004). Bicycle: The History. New Haven et Londres, Yale University Press.

Hill, J. (2006). « Annecdotal evidence : sport, the newspaper press,and history », in Phillips, M.G., Deconstructing Sport History : A Postmodern Analysis. Albant, State University of New York Press, pp. 117-29.

Holt, R. (2014). « Historians and the history of sport », Sport in History, vol. 34, $\mathrm{n}^{\circ}$ 1, pp. 1-33.

Kalifa D., Régnier, P., Thérenty, M.-È., et Vaillant, A. (2011). «Introduction », in Régnier, P., Thérenty, M.-È., et Vaillant, A., La Civilisation du journal : histoire culturelle et littéraire de la presse française au XIXe siècle, Paris : Nouveau Monde
Éditions, pp. 7-21.

Lewis, R.W. (2016). The Stadium Century : Sport, Spectatorship and Mass Society in Modern France. Manchester, Manchester University Press.

Loué, T. (2002). « Un modèle matricial : les revues de culture générale » in Pluet-Despatin, J. Leymaire, M. et Mollier, J-Y, La Belle Époque des revues. Condé-sur-Noireau, Éditions de l'IMEC, pp. 57-68.

Nye, R. (1984). Crime, Madness and Politics in Modern France. Princeton University Press.

Poyer, A. (2004). « La structuration fédérale du cyclisme associatif du Nord et du Pas-de-Calais entre 1881 et 1914 : priorité à la région ou à la nation ? », Revue du Nord, $\mathrm{n}^{\circ} 355$, pp. 297-310.

Smethurst, P. (2015). The Bicycle : Towards A Global History. Basingstoke, Palgrave Macmillan.

Tétart, P. (2015). «Introduction : Des articulets aux pages sportives : état des lieux et des intentions » in Tétart, P., $L a$ presse régionale et le sport : naissance de l'information sportive. Rennes, Presses Universitaire de Rennes, pp. 9-28. Consulté en ligne à https://books.openedition.org/pur/91741 le 5 mai 2021

Thompson, C. (2006). The Tour de France : A Cultural History. Berkeley, Los Angeles et Londres, University of California Press.

Vallet, O. (1998). « Vélo, bicyclette : histoire des mots », Les cahiers de médiologie, 5, pp. 15-18. 


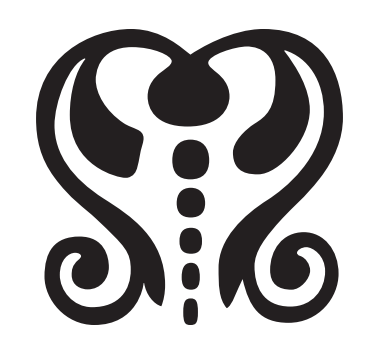




\title{
RÉSumé | Abstract | Resumo
}

\author{
Le Véloce-Sport et l'invention de la culture cycliste \\ The Véloce-Sport and the invention of cycling culture \\ O Veloce-Sport e a invenção da cultura do ciclismo
}

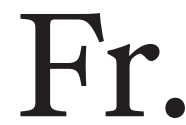

Publié entre 1885 et 1897, Le Véloce-Sport de Bordeaux faisait figure de chef de proue parmi les revues vélocipédiques françaises de la fin du XIX siècle. En tant que la voix - de l'Union Vélocipédique Française (UVF), cet hebdomadaire en devint par la suite son organe national officiel. Alors que certaines analyses de l'histoire du sport en France se sont penchées sur ce rapport avec l'UVF pour mieux comprendre l'évolution de cette dernière, on a plutôt négligé l'analyse des pratiques journalistiques chez Le Véloce-Sport. Une lecture plus attentive de ce dernier offre le spectacle d'une culture sportive en voie de développement et illustre donc le rôle fondamental du journalisme sportif, ainsi que celui de ses lecteurs, dans la construction de cette culture. Notre analyse se portera sur la rubrique « À travers route » et sur les articles publiés sous celle-ci entre 1885 et 1889. À la différence de la plupart d'autres rubriques, « À travers route » encourage le lecteur-cycliste de devenir cycliste-écrivain en lui invitant de raconter ses périples à vélo ; elle permet donc de mieux comprendre la contribution du cycliste-écrivain à la conceptualisation et à la représentation de la vélocipédie ainsi qu'à une culture cycliste nationale en voie de développement. Notre analyse se portera sur deux aspects de ce phénomène qu'on trouve dans les récits des écrivains-cyclistes : la représentation du temps et de l'espace traversés à vélo et l'élaboration du personnage qui les traverse (le véloceman). Écrire le cyclisme devient ainsi une façon d'afficher son appartenance à cette culture cycliste émergeante en France de l'époque tout en contribuant à sa construction et à son élaboration.

Mots-clés : cyclisme ; presse sportive ; XIXème siècle ; culture sportive ; France

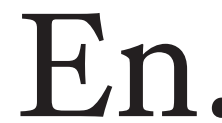

Published between 1885 and 1897, the Bordeaux-based Le Véloce-Sport was a leading figure amongst French cycling reviews of the late nineteenth century. As the came its official national publication. While some sport historians have highlighted its relationship with the UVF in order to better understand the latter's development, scholars have largely failed to analyse the journalistic practices of Le Véloce-Sport itself. A closer reading of the latter offers the spectacle of a developing sports culture and therefore illustrates the fundamental role of sports journalism, as well as the part played by its readers, in the construction of this culture. This study will focus on one section from the review ( « À travers route » [Along the road]) and the articles published in it between 1885 and 1889. Unlike most other sections of Le Véloce-Sport, « À travers route » encourages the cyclist-reader to become a cyclist-writer by inviting the latter to recount his cycling adventures. It thus allows us to better understand the contribution of a broad range of cycling-writers to the conceptualisation and representation of cycling and to a developing national cycling culture. Our analysis will focus on two aspects of this phenomenon through the examination of the submissions by the readers of Le Véloce-Sport: the representation of time and space traversed whilst cycling and the elaboration of the character who traverses these (the véloceman). Writing about cycling thus becomes a means of signalling one's adherence to the emerging cycling culture of the time whilst helping to build and elaborate it.

Keywords: cycling; sports press; 19th century; sports culture; France 

Publicada entre 1885 e 1897, Le Véloce-Sport de Bordeaux impôs-se como a principal revista velocipédica francesa do final do século XIX. Enquanto voz da União Velocipédica Francesa (UVF), o hebdomadário viria a se tornar, mais tarde, seu órgão nacional oficial. Enquanto alguns estudos da história do esporte na França se concentraram nessa relação com a UVF para apreender sua evolução, a análise das próprias práticas jornalísticas na $L e$ Véloce-Sport tem sido negligenciada. Uma leitura mais atenta dessa revista revela o espetáculo de uma cultura esportiva em desenvolvimento, ilustrando o papel fundamental do jornalismo esportivo, e de seus leitores, na construção dessa cultura. Analisamos aqui a coluna "À travers route" e os artigos nela publicados entre 1885 e 1889. Ao contrário da maioria das outras colunas, "À travers route" incentiva o leitor-ciclista a se tornar um ciclista-escritor, convidando-o a contar suas aventuras de bicicleta, proporcionando uma melhor compreensão da contribuição do ciclista-escritor para a conceituação e representação da velocipédica e para uma cultura ciclística francesa em desenvolvimento. Nossa análise foca mais especificamente em dois aspectos desse fenômeno nas narrativas dos escritores-escritores: a representação do tempo e do espaço percorridos de bicicleta; e a elaboração do personagem que os atravessa (o véloceman ou "homem-velocidade"). Escrever sobre ciclismo torna-se assim uma forma de assumir e de manifestar seu pertencimento à cultura do ciclismo emergente na França da época, ao mesmo tempo em que contribui para sua construção e seu desenvolvimento.

Palavras-chave: ciclismo; imprensa esportiva; século XIX; cultura esportiva; França 